\title{
Homotopy Continuation for Correction Procedure via Reconstruction - Discontinuous Galerkin (CPR-DG) Methods
}

\author{
Meilin $\mathrm{Yu}^{1}(\square)$ \\ University of Maryland, Baltimore County, Baltimore, MD 21250 \\ and \\ Z. J. Wang ${ }^{2}$ \\ The University of Kansas, Lawrence, KS 66045
}

\begin{abstract}
Homotopy continuation for correction procedure via reconstruction - discontinuous Galerkin (CPR-DG) methods is developed for solving steady state hyperbolic conservation laws. The efficacy of homotopy continuation has been demonstrated using both 1D and 2D steady flow problems. According to the test results, it is found that homotopy continuation can work robustly on flow simulations of shock capturing for a wide range of grid sizes and polynomial orders without parameter tuning.
\end{abstract}

\section{Introduction}

$\mathrm{T}$ he last two decades have witnessed considerable progress in the development of high-order accurate computational fluid dynamics (CFD) methods. The recent $1^{\text {st }}$ and $2^{\text {nd }}$ International Workshop on High-Order CFD Methods [1] has demonstrated the superior numerical properties of high-order methods compared with traditional low-order schemes with substantial evidence, and pointed out future directions for even wider application of high-order methods in industrial design process.

As has been realized by many researchers [1,2], one big challenge in the development of high-order method is to design a robust, scalable and efficient algorithm for solving the nonlinear system which governs complex fluid flow, e.g., high Reynolds number turbulent flow over complex geometry. For steady flow simulation, mature convergence acceleration techniques $[3,4,5,6,7]$ have been established for low-order finite volume methods. However, these techniques cannot be directly carried over to highorder schemes [2]. Much effort $[8,9,10,11,12,13,14]$ has been spent on developing robust and efficient solution algorithm for high-order schemes.

To solve steady state problems, much attention has been focused on pseudo-transient continuation of the original steady state problem. The argument behind pseudo-transient continuation is that for a steady state problem, the transient components in the flow field will gradually decay as time marches towards infinity [15]. Clearly, pseudo-transient continuation can provide a physical path for propagating boundary information mildly into the whole flow field, especially at the initial stage of a simulation. However, the starting CFL number and the strategy to advance it might vary in practical application. Sometimes, especially for stiff flow problems, e.g., high Reynolds number turbulent flow, high-speed flow with shocks, etc., convergence (i.e., machine zero) cannot be achieved when pseudo-transient continuation is used. This can dramatically hinder the application of high-order methods in industrial design process.

Homotopy continuation, which has been widely adopted in numerical algebraic geometry [16] and bifurcation analysis [17, 18], provides an alternative way for solving the nonlinear system governing fluid flow. Recently works on the development of homotopy continuation methods in CFD include the application for finite difference schemes [19, 20, 21] and WENO schemes [22]. In contrast to the pseudotransient continuation, the basic idea behind homotopy continuation is to gradually deform the flow field from an easy-to-solve stage to the final stage governed by the original steady state problem. The

\footnotetext{
${ }^{1}$ Assistant Professor, Department of Mechanical Engineering, AIAA Member, email: $\underline{\text { mlyu @ umbc.edu }}$

${ }^{2}$ Spahr Professor and Chair, Department of Aerospace Engineering, AIAA Associate Fellow, email: zjw@ ku.edu 1 of 17

American Institute of Aeronautics and Astronautics
} 
advantage of homotopy continuation is that it is independent of CFL number, and is robust for a wide range of flow conditions. In this paper, we focus on the development of homotopy continuation for flux reconstruction (FR)/correction procedure via reconstruction (CPR) methods.

FR/CPR [23, 24, 25] is a recently developed high-order accurate numerical algorithm for conservation laws. It has been proven that FR/CPR can generalize several popular high-order accurate methods [23, 26, 27, 28, 29], including discontinuous Galerkin (DG) [30], spectral difference (SD) [31, 32] and spectral volume (SV) [33]. In this study, the CPR scheme which is equivalent to DG (also named as CPR-DG) is adopted. Clearly, the same homotopy continuation approach can be easily adapted to DG, SD and SV. For more details about the recent development of CPR, readers are referred to the review paper [34].

The organization of the paper is as follows. For the sake of completeness, we briefly review FR/CPR methods for steady state problems in Section II. Introduction of numerical continuation, including both pseudo-transient continuation and homotopy continuation, is presented in Section III. The localized artificial viscosity method is also introduced in Section III. In Section IV, numerical examples are shown to demonstrate the efficacy of homotopy continuation, and to compare the numerical performance of homotopy continuation and pseudo-transient continuation. Finally, conclusions are summarized in Section V.

\section{Correction Procedure via Reconstruction (CPR)}

The hyperbolic conservation law in conservative form can be written as

$$
\frac{\partial Q}{\partial t}+\sum_{l=1}^{d} \frac{\partial f^{l}}{\partial x^{l}}(Q)=0
$$

where $Q$ is the vector of conserved variables, $f^{l}$ is the flux in the $x^{l}$ direction, and $d$ is the dimension. Note that for inviscid flow, the hyperbolic conservation law becomes Euler equations with $Q=$ $(\rho, \rho u, \rho v, E)$, where $\rho$ is the fluid density, $u$ and $v$ are the Cartesian velocity components, and $E$ is the total internal energy. On setting $\partial Q / \partial t \equiv 0$, the corresponding steady state problem reads

$$
\sum_{l=1}^{d} \frac{\partial f^{l}}{\partial x^{l}}(Q)=0
$$

Eq. (2) is defined on the spatial domain $\Omega$ bounded by $\partial \Omega$. To solve the boundary value problem numerically, FR/CPR is used to discretize Eq. (2). It is assumed that the physical domain $\Omega$ is partitioned into $\mathrm{N}$ non-overlapping elements, denoted as $\Omega^{i}$. The FR/CPR discretization can then be developed on each element $\Omega^{i}$ as follows.

Consider the weak form of Eq. (2) on $\Omega^{i}$ with the solution approximation $Q_{h}$,

$$
\int_{\Omega^{i}} W \sum_{l=1}^{d} \frac{\partial f^{l}}{\partial x^{l}}\left(Q_{h}\right) d V=0,
$$

where $W$ is the test function, and belongs to the same space as $Q_{h}$ in the CPR-DG scheme. In this study, $Q_{h}$ is selected from the polynomial space or the tensor product of the polynomial space. On integration by parts, one can rewrite Eq. (3) as

$$
-\int_{\Omega^{i}} \sum_{l=1}^{d} \frac{\partial W}{\partial x^{l}} f^{l}\left(Q_{h}\right) d V+\int_{\partial \Omega^{i}} W f^{n} d S=0,
$$

where $f^{n}=\sum_{l=1}^{d} f^{l} n^{l}$, is the normal flux, and $n^{l}$ is the component of the unit surface normal vector $\boldsymbol{n}$ in the $x^{l}$ direction.

In order to ensure conservation, normal fluxes $f^{n}$ on the surface $\partial \Omega^{i}$ shared by element $\Omega^{i}$ and its neighbors $\Omega^{i+}$ need to be assigned common values. For the hyperbolic conservation law, an approximate 
Riemann solver can be used to calculate the common fluxes. In this work, the Roe Riemann solver [35] is adopted. Substituting $f^{n}$ with the corresponding common values, we reformulate Eq. (4) as

$$
-\int_{\Omega^{i}} \sum_{l=1}^{d} \frac{\partial W}{\partial x^{l}} f^{l}\left(Q_{h}\right) d V+\int_{\partial \Omega^{i}} W f_{c o m}^{n}\left(Q_{h}^{i}, Q_{h}^{i+}, \boldsymbol{n}\right) d S=0 .
$$

On using integration by parts again on the weak form Eq. (5), one obtains the strong form as follows

$$
\int_{\Omega^{i}} W \sum_{l=1}^{d} \frac{\partial f^{l}}{\partial x^{l}}\left(Q_{h}\right) d V+\int_{\partial \Omega^{i}} W\left(f_{\text {com }}^{n}-f^{n}\right) d S=0,
$$

In order to eliminate the test function $W$, the surface integral in Eq. (6) is cast into the form of a volume integral via the introduction of a correction field $\delta^{C}$ on the element $\Omega^{i}$, which belongs to the same space as that of the solution $Q_{h}$. This is expressed as

$$
\int_{\Omega^{i}} W \delta^{C} d V=\int_{\partial \Omega^{i}} W\left(F_{c o m}^{n}-F^{n}\right) d S,
$$

On substituting Eq. (7) into Eq. (6), one obtains

$$
\int_{\Omega^{i}} W\left(\sum_{l=1}^{d} \frac{\partial f^{l}}{\partial x^{l}}\left(Q_{h}\right)+\delta^{C}\right) d V=0 .
$$

If $f^{l}$ is a linear function of $Q_{h}$, then $\sum_{l=1}^{d} \partial f^{l} / \partial x^{l}$ sits in the same space as that of $Q_{h}$. In this case, the terms enclosed by the parentheses are approximated by the same space. For nonlinear conservation laws, $\sum_{l=1}^{d} \partial f^{l} / \partial x^{l}$ is generally not an element of the polynomial space or the tensor product of the polynomial space. In this case, the most obviously choice is to project $\sum_{l=1}^{d} \partial f^{l} / \partial x^{l}$ onto the same space as that of $Q_{h}$. This is written as

$$
\int_{\Omega^{i}} W \prod\left(\sum_{l=1}^{d} \frac{\partial f^{l}}{\partial x^{l}}\left(Q_{h}\right)\right) d V=\int_{\Omega^{i}} W\left(\sum_{l=1}^{d} \frac{\partial f^{l}}{\partial x^{l}}\left(Q_{h}\right)\right) d V .
$$

Then Eq. (8) reduces to

$$
\Pi\left(\sum_{l=1}^{d} \frac{\partial f^{l}}{\partial x^{l}}\left(Q_{h}\right)\right)+\delta^{C}=0
$$

Two efficient approaches, namely the Lagrange polynomial (LP) approach and the chain-rule (CR) approach, can be used to calculate the projection of the flux divergence. More details can be found in Ref. [29]. This completes the formulation of the FR/CPR discretization.

\section{Numerical Methods}

\section{A. Numerical Continuation Methods}

By substituting the left-hand side of Eq. (10) with $-R\left(Q_{h}\right)$, the general form of Eq. (10) can be written as

$$
R\left(Q_{h}\right)=0 .
$$

Generally, $R\left(Q_{h}\right)$ is a nonlinear system with respect to $Q_{h}$. For conciseness, $Q_{h}$ is replaced with $Q$ in the rest of the paper, if no confusion between $Q_{h}$ and $Q$ arises.

To solve Eq. (11), a common approach is to linearize it around the solution $Q^{n}$ at the $n_{t h}$ step, and assume that $R\left(Q^{n+1}\right) \approx 0$. This is de facto Newton's method, which is expressed as

$$
-\frac{\partial R}{\partial Q}\left(Q^{n}\right) \Delta Q^{(n)}=R\left(Q^{n}\right),
$$

3 of 17

American Institute of Aeronautics and Astronautics 
where $\Delta Q^{(n)}=Q^{n+1}-Q^{n}$. The convergence of Newton method can be very fast (i.e., quadratic convergence rate) when the initial guess is sufficiently close to the solution. However, it is not a trivial task to estimate a suitable initial iterate for general CFD simulations. Therefore, numerical continuations methods are used to provide reasonably good initial guess for Newton method. Two types of numerical continuation methods, namely the pseudo-transient continuation and the homotopy continuation, are introduced in this section.

\section{A.1. Pseudo-transient Continuation}

Time marching methods originally developed for unsteady flow simulation can be used as a relaxation on Newton's method. For a steady problem, it is expected that the transient components in the flow fields can be gradually damped as time marches. As a result, the pseudo-transient continuation might provide a suitable initial guess for Newton's method. In this approach, Eq. (12) can be rewritten as

$$
\left(\frac{I}{(\Delta t)^{n}}-\frac{\partial R}{\partial Q}\left(Q^{n}, t^{n}\right)\right) \Delta Q^{(n)}=R\left(Q^{n}, t^{n}\right)
$$

where $(\Delta t)^{n}$ is the time step size at the $n_{t h}$ step. Note that when $(\Delta t)^{n}$ is small enough, Eq. (13) is a backward Euler discretization of the unsteady equation $\partial Q / \partial t=R(Q)$; when $(\Delta t)^{n}$ becomes positive infinity, Newton's method is recovered. As stated previously, the pseudo-transient continuation does not always guarantee a suitable initial guess for Newton's method. The starting time step size and the strategy to advance $\Delta t$ may vary case by case.

\section{A.2. Homotopy Continuation}

The homotopy continuation provides an alternative way for solving the steady state problem Eq. (11) using Newton's method. We use $t \in[0,1]$ to denote the homotopy parameter, and formulate the fixedpoint homotopy as

$$
H(Q, t)=(1-t)\left(R(Q)-\beta t \nabla^{2} Q\right)+t\left(Q-Q^{0}\right)=0,
$$

where $Q^{0}$ is the initial condition which could be a solution of $R(Q)=0$ with trivial boundary conditions, and $\beta$ is a free parameter. It is noted that when $t=0$, the original steady state problem is recovered; when $t=1, Q=Q^{0}$ is naturally satisfied by design.

Newton's method is used to solve Eq. (14). Similar to Eq. (12), the linearization of Eq. (14) around $\left(Q^{n}, t^{n}\right)$ can be written as

$$
H\left(Q^{n}, t^{n}\right)+\frac{\partial H}{\partial Q}\left(Q^{n}, t^{n}\right) \Delta Q^{(n)}+\frac{\partial H}{\partial t}\left(Q^{n}, t^{n}\right) \Delta t^{(n)}=0 .
$$

Herein, $\Delta t^{(n)}=t^{n+1}-t^{n}$ is the homotopy step size.

The predictor-corrector procedure is usually used to solve Eq. (15). The form of Eq. (15) has been slightly modified to enhance convergence while still maintaining similar computational cost in the predictor-corrector procedure. Specifically, the predictor phase can be written as

$$
\frac{\partial H}{\partial Q}\left(Q^{(n-1)^{*}}, t^{n}\right)\left(Q^{n^{*}}-Q^{n}\right)=-\frac{\partial H}{\partial t}\left(Q^{n}, t^{n}\right) \Delta t^{(n)},
$$

where the superscript ' $n$ "' stands for the latest state in the $n_{t h}$ step. The correction phase is expressed as

$$
\frac{\partial H}{\partial Q}\left(Q^{n^{*}}, t^{n+1}\right)\left(Q^{n+1}-Q^{n^{*}}\right)=-H\left(Q^{n^{*}}, t^{n+1}\right) \text {. }
$$

We note that in the proposed predictor-corrector procedure, only one Jacobian calculation, i.e., $\frac{\partial H}{\partial Q}\left(Q^{n^{*}}, t^{n+1}\right)$ in the corrector phase, is needed. The Jacobian in the predictor phase of the $n_{t h}$ step is actually that calculated in the corrector phase of the $(n-1)_{t h}$ step. 


\section{B. Localized Laplacian Artificial Viscosity Method}

Shock capturing remains challenging for high-order accurate methods. The steady flow problem is usually hard to converge when shock exists. Therefore, homotopy continuation is adopted in this study to facilitate convergence. To stabilize shock, the localized artificial viscosity method is used. This method is introduced as follows.

Consider the Euler equation augmented with a general artificial diffusion term:

$$
\frac{\partial Q}{\partial t}+\sum_{l=1}^{d} \frac{\partial f^{i n v, l}}{\partial x^{l}}(Q)=\sum_{l=1}^{d} \frac{\partial f^{v i s, l}}{\partial x^{l}}(Q, \nabla Q) .
$$

In the localized Laplacian artificial viscosity (LLAV) method, the artificial diffusive terms $\frac{\partial f^{v i s, l}}{\partial x^{l}}(Q, \nabla Q), l=1, \ldots, d$ is of Laplacian type, which reads $\sum_{l=1}^{d} \frac{\partial}{\partial x^{l}}\left(\varepsilon \frac{\partial Q}{\partial x^{l}}\right)$. Then Eq. (18) is modified as

$$
\frac{\partial Q}{\partial t}+\sum_{l=1}^{d} \frac{\partial f^{i n v, l}}{\partial x^{l}}(Q)=\sum_{l=1}^{d} \frac{\partial}{\partial x^{l}}\left(\varepsilon \frac{\partial Q}{\partial x^{l}}\right) .
$$

For each element e, the element-wise artificial viscosity $\varepsilon_{\mathrm{e}}$ can be modeled as [36]

$$
\varepsilon_{e}=\left\{\begin{array}{cl}
\frac{\varepsilon_{0}}{2}\left(1+\sin \frac{\pi\left(S_{e}-S_{0}\right)}{2 \kappa}\right) & \begin{array}{l}
\text { if } S_{e}<S_{0}-\kappa \\
\text { if } S_{0}-\kappa \leq S_{e} \leq S_{0}+\kappa . \\
\varepsilon_{0}
\end{array} \text { if } S_{e}>S_{0}+\kappa .
\end{array}\right.
$$

Herein, $\mathrm{S}_{\mathrm{e}}$ is the smoothness indicator; $\mathrm{S}_{0}$ is a reference value of the smoothness indicator for smooth flow features with degree $\mathrm{k}$ reconstruction, which is modeled as $-3 \log _{10}(\mathrm{k})$ in this study; $\kappa$ is a free parameter to control the functioning spectra of the artificial dissipation, which is fixed at 4.0 in this study; and $\varepsilon_{0}$ is the amount of artificial viscosity added to the solution.

The smoothness indicator $S_{e}$ in Eq. (20) is reconstructed as follows. First, the degree k reconstruction of the solution can be written as

$$
Q_{h}=\sum_{j=1}^{N(k)} Q_{h, j} L_{j}
$$

where $L_{j}$ is the $j$-th basis function from the degree k polynomial space on $\Omega^{i}$, and $N(k)$ is the total number of bases. Then, the solution $Q_{h}$ is projected onto the degree $\mathrm{k}-1$ polynomial space on $\Omega^{\mathrm{i}}$. This is expressed as

$$
Q_{h}^{\text {proj }}=\sum_{j=1}^{N(k-1)} \hat{Q}_{h, j} \hat{L}_{j},
$$

where $\hat{\mathrm{L}}_{\mathrm{j}}$ and $\mathrm{N}(\mathrm{k}-1)$ are the $\mathrm{j}$-th basis function and the total number of bases for the degree $\mathrm{k}-1$ polynomial space on $\Omega^{\mathrm{i}}$, respectively. The expansion coefficients $\widehat{Q}_{h, j}$ can be calculated by solving the following linear system,

$$
\sum_{j=1}^{N(k-1)} \hat{Q}_{h, j}\left\langle\hat{L}_{j}, \hat{L}_{m}\right\rangle=\sum_{j=1}^{N(k)} Q_{h, j}\left\langle L_{j}, \hat{L}_{m}\right\rangle, \quad m=1, \cdots, N(k-1) .
$$

Note that $\langle\cdot, \cdot\rangle$ indicates the inner product in $L_{2}\left(\Omega^{i}\right)$.

The resolution-based smoothness indicator $\mathrm{S}_{\mathrm{e}}$ is then defined as

$$
S_{e}=\log _{10} \frac{\left\langle Q_{h}-Q_{h}^{\text {proj }}, Q_{h}-Q_{h}^{\text {proj }}\right\rangle_{e}}{\left\langle Q_{h}, Q_{h}\right\rangle_{e}} \text {. }
$$


We note that this smoothness indicator can robustly detect flow discontinuities, as $S_{e}$ is much larger than $S_{0}$ when the flow field is not smooth.

The amount of artificial viscosity added to the solution $\varepsilon_{0}$ can be modeled as a function of the Péclet number Pe, the subcell resolution $\Delta \xi_{\max }$ for a degree $\mathrm{k}$ reconstruction on a mesh of grid size $\mathrm{h}$, and the characteristic speed in the flow field, e.g., the maximum eigenvalue of the Euler system $|\lambda|_{\text {max }}$ :

$$
\varepsilon_{0}=f\left(\Delta \xi_{\max }, P e\right) \cdot h \cdot|\lambda|_{\text {max }} .
$$

The linear function $f\left(\Delta \xi_{\text {max }}, P e\right)$ in Eq. (25) used in this study is modeled as

$$
f\left(\Delta \xi_{\text {max }}, P e\right)=-\frac{\Delta \xi_{\max }}{P e}+\frac{2}{P e} .
$$

More modelling details regarding the localized Laplacian artificial viscosity method, and the verification and validation of the method can be found in Refs. [37, 38].

\section{Solution Strategy of the Linear System}

Both an in-house generalized minimal residual method (GMRES) linear system solver and the GMRES solver in the Portable, Extensible Toolkit for Scientific Computation (PETSc) library are used to solve the large linear systems derived from pseudo-transient or homotopy continuation. As is known, the quality of preconditioners is essential for the performance of the GMRES solver. In the in-house GMRES solver, the lower-upper symmetric-Gauss-Seidel (LUSGS) method is used as the preconditioner. In PETSc, the choices of preconditioners are flexible. In this study, it is found that the ILU(0) preconditioner is efficient for all test problems. Therefore, the ILU(0) preconditioner is adopted in all simulations performed using the PETSc library.

\section{Numerical Results and Discussions}

In this section, the efficacy of homotopy continuation is tested for both 1D and 2D flow simulations. First of all, the performance of homotopy continuation and pseudo-transient continuation are compared using 1D subsonic and transonic flows. Then the homotopy implementation for $2 \mathrm{D}$ flow simulations is validated using the manufactured solution [39]. After that, the homotopy continuation method is used to simulate the steady oblique shock reflection problem.

\section{A. 1D Nozzle Flows}

A 1D convergent-divergent nozzle is used in this section to demonstrate the efficacy of pseudotransient and homotopy continuation methods. The nozzle shape is specified as follows,

$$
\left\{\begin{array}{l}
A=1.0-0.661514 * e^{-(\log 2) * x^{2}}, x \in[-4,0) \\
A=0.536572-0.198086 * e^{-(\log 2) * x^{2}}, x \in[0,4]
\end{array} .\right.
$$

Both subsonic and transonic flows are simulated. For the subsonic case, the inlet Mach number, density and pressure are set as $0.2,1.4$ and $1.0 / \gamma$, respectively. Then based on the geometry and isentropic flow properties, the exit flow conditions can be calculated. For the transonic case, the shock position is located at $x=1.5$, and the inlet density and pressure are set as 1.4 and $1.0 / \gamma$, respectively. Then based on the Rankine-Hugoniot conditions at the shock and sonic throat conditions, other boundary flow conditions can be calculated. For simplicity, all flow conditions are specified weakly on both inlet and outlet boundaries via Riemann solvers. The flow fields, including density, pressure and Mach number, for both subsonic and transonic cases are displayed in Fig. 1.

\section{A.1. Subsonic Nozzle Flow}

For subsonic flow simulation, the numerical cost of different algorithms for solving Eq. (11) is compared. These algorithms include Newton's method (i.e., Eq. (12)), pseudo-transient continuation (i.e., Eq. (13)), homotopy continuation (i.e., Eq. (14)), and explicit Runge-Kutta (RK) time marching. For the explicit time marching, the CFL number is set as $1 /(2 k+1)$, which is suitable for DG discretizations 
using polynomials of degree $k$ and a $k+1$ stage RK method of order $k+1$. For pseudo-transient continuation, the initial CFL number is also set as $1 /(2 k+1)$, and increases as a function of the $L_{2}$ norm $\left\|R\left(Q^{n}\right) / R\left(Q^{0}\right)\right\|$. In pseudo-transient continuation, for a certain convergence threshold $\varepsilon_{\text {conv }}$, when $\left\|R\left(Q^{n}\right) / R\left(Q^{0}\right)\right\|<\varepsilon_{\text {conv }}$, the CFL number will be set as infinity. Newton's method is then recovered with a good initial guess.

The computational cost comparison on different meshes using $P^{2}, P^{3}$ and $P^{4}$ reconstructions are presented in Fig. 2. The work unit is a non-dimensional measure of the CPU time defined in Ref. [29]. To facilitate comparison, the work unit is further normalized by a reference value. In this case, the reference value is set as the work unit of the homotopy continuation using $P^{2}$ reconstruction on the mesh with 10 elements. It is observed that both Newton's method and pseudo-transient continuation work very efficiently for this case. Homotopy continuation is less efficient than Newton's method and pseudotransient continuation, but much more efficient than the explicit method. The reason why homotopy continuation has inferior performance than that of Newton's method and pseudo-transient continuation can be explained as follows.

For the subsonic case, the free stream condition is already a good initial guess for Newton's method. In homotopy continuation, large amount of CPU time is spent on solving the linear systems on the homotopy tracking path. Therefore, Newton's method and pseudo-transient continuation can outperform homotopy. The converging paths of both homotopy continuation and pseudo-transient continuation are displayed in Fig. 3. It is observed that during convergence, the intermediate flow fields for homotopy continuation are smooth. But the intermediate flow fields for pseudo-transient continuation exhibit certain oscillation.

\section{A.2. Transonic Nozzle Flow}

For transonic flow simulation, the localized Laplacian artificial viscosity method is used to stabilize the CPR method. The CFL number for the explicit RK scheme is severely penalized by the artificial diffusive terms [40]. Therefore, simulations are not carried out using the explicit RK scheme. Due to the flow discontinuity, free stream conditions are not good initial guess for Newton's method. As a result, we use numerical continuation to provide suitable initial guess for Newton's method.

The numerical cost of homotopy continuation and pseudo-transient continuation is compared in Fig. 4 on different meshes using $P^{2}, P^{3}$ and $P^{4}$ reconstructions. Note that the parameters in the localized artificial viscosity are fixed for all simulations, as stated previously in Section III.2. It is observed that pseudo-transient continuation can only function well in a certain reconstruction range as shown in Fig. 4. However, homotopy continuation works robustly for a wide range of grid sizes and polynomial orders. The converging paths of both homotopy continuation and pseudo-transient continuation are displayed in Fig. 5.

\section{B. 2D Flow Problems}

From 1D tests, it is found that homotopy continuation is a viable alternative to pseudo-transient continuation. It can robustly work on shock capturing for a wide range of grid sizes and polynomial orders without parameter tuning. Thus homotopy continuation is extended to simulate $2 \mathrm{D}$ flow problems.

\section{B.1. 2D Manufactured Solution}

To test the implementation of the homotopy implementation for $2 \mathrm{D}$ flow simulations, the manufactured solution approach [39] is adopted in this section. Source terms are added to Eq. (1), which states

$$
\frac{\partial Q}{\partial t}+\sum_{l=1}^{d} \frac{\partial f^{l}}{\partial x^{l}}(Q, \nabla Q)=S(Q)
$$

Herein, the source term is calculated from the following conditions, 


$$
\left\{\begin{array}{l}
\rho=0.5\left(\sin \left(x^{2}+y^{2}\right)+1.5\right) \\
u=\sin \left(x^{2}+y^{2}\right)+1.5 \\
v=0.1\left(\cos \left(x^{2}+y^{2}\right)+0.5\right) \\
E=0.5\left(\cos \left(x^{2}+y^{2}\right)+1.5\right)
\end{array}\right.
$$

The computational domain is set as $[-0.1,0.2] \times[0,0.5]$. The converged solution for the density $\rho$ using $P^{3}$ reconstruction on a $40 \times 72$ mesh is displayed in Fig. 6 (a). The order of accuracy of the spatial discretization is tested for $P^{2}, P^{3}$ and $P^{4}$ reconstruction on a set of meshes, tessellated with $5 \times 9$, $10 \times 18,20 \times 36,40 \times 72$ and $80 \times 144$ elements, respectively. The results of order of accuracy study are shown in Fig. 6 (b). It is clear that the optimal convergence rate is achieved for different reconstruction orders. The initial condition $Q^{0}$ in homotopy continuation is set as a constant vector at $x=0$ and $y=0$, which is $(\rho, u, v, E)=(0.75,1.5,0.15,1.25)$. This is a trivial solution for the Euler equation without source terms. It is found that this initial condition works well for all simulations presented in this section.

\section{B.2. Oblique Shock Reflection}

In this section, a 2D oblique shock reflection case is used to test the efficacy of homotopy continuation on shock-capturing related simulations. This test case describes an oblique shock wave reflection on an inviscid wall. The incident shock of Mach 3 has a $33^{\circ}$ angle with respect to the $\mathrm{x}$-axis. The computational domain is $[-1.5,2] \times[0,1.5]$. Three sets of meshes with characteristic element lengths of $1 / 10,1 / 20$ and $1 / 40$ are used in this study. All simulations have converged 12 orders (i.e., the residual is decreased from 1.0 to $10^{-12}$ ). Note that the parameters in the localized artificial viscosity are fixed at the same values as those in Section IV.A.2 for all simulations presented in this section.

Density and artificial viscosity contours using $P^{2}$ reconstruction on three sets of meshes are presented in Fig. 7. Density contours using different reconstruction orders, namely, degree 2 to 4 , on the medium mesh are shown in Fig. 8. It is clear that when the reconstruction order or the mesh is refined, LLAV can capture shock waves more sharply.

The homotopy tracking paths for different reconstruction orders and meshes are presented in Fig. 9 (a). We found that for all simulations, an almost constant number of tracking steps (e.g., about 12 steps for all the tests in this section) are needed to decrease homotopy from 1 to 0 . The normalized work unit versus degrees of freedoms (DOFs) of different orders of reconstruction is shown in Fig. 9 (b). It is concluded that the computational cost almost linearly increase as DOFs increase for different orders of reconstruction.

\section{Conclusions}

Homotopy continuation for CPR-DG methods is developed to solve steady hyperbolic conservation law. The efficacy of homotopy continuation has been demonstrated using both 1D and 2D steady flow problems. Numerical results from 1D nozzle flow simulation for both homotopy continuation and pseudotransient continuation are compared. It is found that for the subsonic flow, pseudo-transient continuation works more efficiently than homotopy continuation in this moderately stiff flow system. But for the transonic flow, the computational cost for both continuation methods is comparable. Both 1D and 2D results indicate that homotopy continuation works robustly on flow simulations of shock capturing for a wide range of grid sizes and polynomial orders without parameter tuning.

\section{References}

[1] Z. J. Wang, K. Fidkowski, R. Abgrall, F. Bassi, D. Caraeni, A. Cary, H. Deconinck, R. Hartmann, K. Hillewaert, H. Huynh, N. Kroll, G. May, P.-O. Persson, B. v. Leer and M. Visbal, "High-order CFD methods: current status and perspective," Int. J. Numer. Meth. Fluids, vol. 00, pp. 1-42, 2012.

[2] G. May and A. Jameson, "Efficient relaxation methods for high-order discretization of steady problems," in 
Adaptive high-order methods in computational fluid dynamics, Z. Wang, Ed., Singapore, World Scientific, 2011, pp. 363-390.

[3] A. Jameson, "Solution of the Euler equations for two dimensional transonic flow by a multigrid method," Appl. Math. Comp., vol. 13, pp. 327-356, 1983.

[4] S. Yoon and A. Jameson, "Lower-upper symmetric-Gauss-Seidel method for the Euler and Navier-Stokes equations," AIAA Journal, vol. 26, no. 9, pp. 1025-1026, 1988.

[5] N. A. Pierce and M. B. Giles, "Preconditioned multigrid methods for compressible flow calculations on stretched meshes," J. Comp. Phys., vol. 136, pp. 425-445, 1997.

[6] D. J. Mavriplis, "Multigrid strategies for viscous flow solvers on anisotropic unstructured meshes," J. Comp. Phys., vol. 145, pp. 141-165, 1998.

[7] R. Chen and Z. Wang, "Fast, block lower-upper symmetric-Gauss-Seidel scheme for arbitrary grids," AIAA Journal, vol. 38, pp. 2238-2245, 2000.

[8] K. J. Fidkowski, T. A. Oliver, J. Lu and D. L. Darmofal, "p-Multigrid solution of high-order discontinuous Galerkin discretizations of the compressible Navier-Stokes equations," J. Comp. Phys., vol. 207, pp. 92-113, 2005.

[9] P. O. Persson and J. Peraire, "Newton-GMRES preconditioning for discontinuous Galerkin discretizations of the Navier-Stokes equations," SIAM J. on Scientific Computing, vol. 30, pp. 2709-2733, 2008.

[10] Y. Z. Sun, Z. J. Wang and Y. Liu, "Efficient implicit non-linear LU-SGS approach for compressible flow computation using high-order spectral difference method," Commun. Comput. Phys., vol. 5, pp. 760-778, 2009.

[11] C. Liang, R. Kannan and Z. Wang, "A p-Multigrid spectral difference method with explicit and implicit smoothers on unstructured triangular grids," Computers \& fluids, vol. 38, pp. 254-265, 2009.

[12] M. Parsani, V. d. Abeele, L. C. K. and E. Turkel, "Implicit LU-SGS algorithm for high-order methods on unstructured grid with p-multigrid strategy for solving the steady Navier-Stokes equations," J. Comp. Phys., vol. 229, pp. 828-850, 2010.

[13] G. May, F. Iacono and A. Jameson, "A hybrid multilevel method for high-order discretization of the Euler equations on unstructured meshes," J. Comp. Phys., vol. 229, pp. 3938-3956, 2010.

[14] M. A. d. B. Ceze, A robust hp-adaptation method for discontinuous Galerkin discretizations applied to aerodynamic flows, Ph.D. Thesis, 2013.

[15] H. Lomax, T. H. Pulliam and D. W. Zingg, Fundamentals of Computational Fluid Dynamics, Springer-Verlag, 2001.

[16] A. Sommese and C. Wampler, Numerical Solution of Systems of Polynomials Arising in Engineering and Science, Singapore: World Scientific, 2005.

[17] H. B. Keller, "Numerical solution of bifurcation and nonlinear eigenvalue problems," in Applications of Bifurcation Theory, New York, Academic Press, 1977, pp. 359-384.

[18] C. Wales, A. L. Gaitonde, D. P. Jones, D. Avitabile and A. R. Champneys, "Numerical continuation of high Reynolds number external flows," International Journal for Numerical Methods in Fluids, vol. 68, pp. 135$159,2012$.

[19] J. E. Hicken and D. W. Zingg, "Globalization strategies for inexact-Newton solvers," in 19th AIAA Computational Fluid Dynamics Conference and Exhibit, San Antonia, Texas, United States, 2009.

[20] J. E. Hicken, H. Buckley, M. Osusky and D. W. Zingg, "Dissipation-based continuation: a globalization for inexact-Newton solvers," in 20th AIAA Computational Fluid Dynamics Conference, Honolulu, Hawaii, United States, 2011, AIAA-2011-3237.

[21] D. Brown and D. W. Zingg, "Advances in homotopy continuation methods in computational fluid dynamics," in 21st AIAA Computational Fluid Dynamics Conference, San Diego, CA, USA, 2013, AIAA-2013-2370.

[22] W. Hao, J. D. Hauenstein, C.-W. Shu, A. J. Sommese, Z. Xu and Y.-T. Zhang, "A homotopy method based on WENO schemes for solving steady state problems of hyperbolic conservation laws," Journal of Computational Physics, vol. 250, pp. 332-346, 2013.

[23] H. T. Huynh, "A flux reconstruction approach to high-order schemes including discontinuous Galerkin methods," AIAA Paper 2007-4079.

[24] H. T. Huynh, "A reconstruction approach to high-order schemes including discontinuous Galerkin for 
diffusion," AIAA Paper 2009-403.

[25] Z. J. Wang and H. Y. Gao, "A unifying lifting collocation penalty formulation including the discontinuous Galerkin, spectral volume/difference methods for conservation laws on mixed grids," J. Comput. Phys., vol. 228, pp. 8161-8186, 2009.

[26] P. E. Vincent, P. Castonguay and A. Jameson, "A new class of high-order energy stable flux reconstruction schemes," J. Sci. Comput., vol. 47, no. 1, pp. 50-72, 2011.

[27] M. L. Yu and Z. J. Wang, "On the connection between the correction and weighting functions in the correction procedure via reconstruction method," J. Sci. Comput., vol. 54, no. 1, pp. 227-244, 2013.

[28] M. L. Yu and Z. J. Wang, "On the accuracy and efficiency of several discontinuous high-order formulations," in the 51st AIAA Aerospace Sciences Meeting Including the New Horizons Forum and Aerospace Exposition, Grapevine (Dallas/Ft. Worth Region), Texas, AIAA-2013-0855, 2013.

[29] M. L. Yu, Z. J. Wang and Y. Liu, "On the accuracy and efficiency of discontinuous Galerkin, spectral difference and correction procedure via reconstruction methods," Journal of Computational Physics, vol. 259, pp. 70-95, 2014.

[30] B. Cockburn and C.-W. Shu, "TVB Runge-Kutta local projection discontinuous Galerkin finite element method for conservation laws II: general framework," Math. Comput., vol. 52, pp. 411-435, 1989.

[31] D. A. Kopriva and J. H. Kolias, "A conservative staggered-grid Chebyshev multi-domain method for compressible flows," J.Comput.Phys., vol. 125, no. 1, pp. 244-261, 1996.

[32] Y. Liu, M. Vinokur and Z. J. Wang, "Spectral difference method for unstructured grids I: Basic formulation," $J$. Comput. Phys, vol. 216, pp. 780-801, 2006.

[33] Z. J. Wang, "Spectral (finite) volume method for conservation laws on unstructured grids: basic formulation," J. Comput. Phys., vol. 178, pp. 210-251, 2002.

[34] H. Huynh, Z. Wang and P. Vincent, "High-order methods for computational fluid dynamics: A brief review of compact differential formulations on unstructured grids," Computers \& Fluids, vol. 98, pp. 209-220, 2014.

[35] P. L. Roe, "Approximate Riemann solvers, parameter vectors and difference schemes," J. Comput. Phys., vol. 43, no. 2, pp. 357-372, 1981.

[36] P.-O. Persson and J. Peraire, "Sub-Cell Shock Capturing for Discontinuous Galerkin Methods," in 44th AIAA Aerospace Sciences Meeting and Exhibit, Reno, Nevada, 2006, AIAA-2006-0112.

[37] M. L. Yu and Z. J. Wang, "Shock capturing for the correction procedure via reconstruction method using artificial viscosity and diffusivity," in the 8th ICCFD Conference, ICCFD8-2014-0079, Chengdu, China, 2014.

[38] J. S. Park, M. L. Yu, C. Kim and Z. J. Wang, "Comparative study of shock-capturing methods for high-order CPR: MLP and artificial viscosity," in the 8th ICCFD Conference, ICCFD8-2014-0067, Chengdu, China, 2014.

[39] R. Glasby, N. Burgess, K. Anderson, L. Wang, D. Mavriplis and S. Allmaras, "Comparison of SU/PG and DG finite-element techniques for the compressible Navier-Stokes equations on anisotropic unstructured meshes," in the 51st AIAA Aerospace Sciences Meeting, AIAA paper 2013-0691, Dallas, TX, 2013.

[40] A. W. Cook, "Artificial fluid properties for large-eddy simulation of compressible turbulent mixing," Physics of Fluids, vol. 19, p. 055103, 2007. 


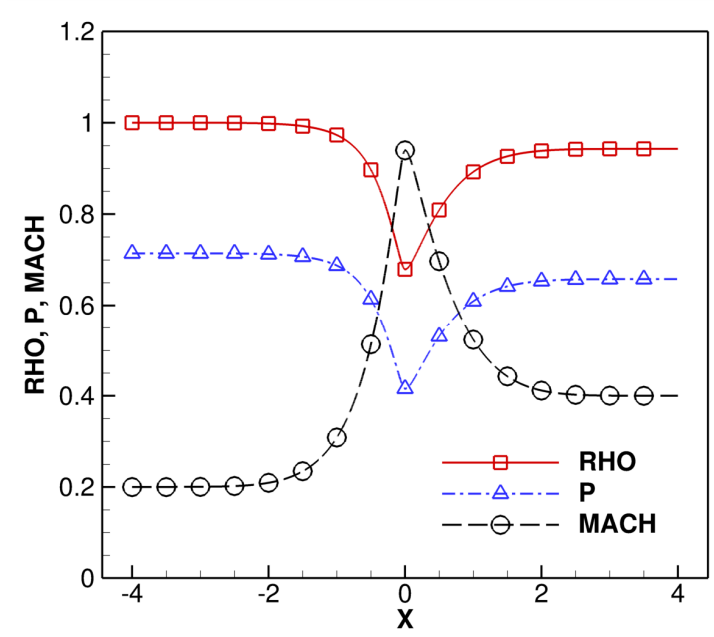

(a)

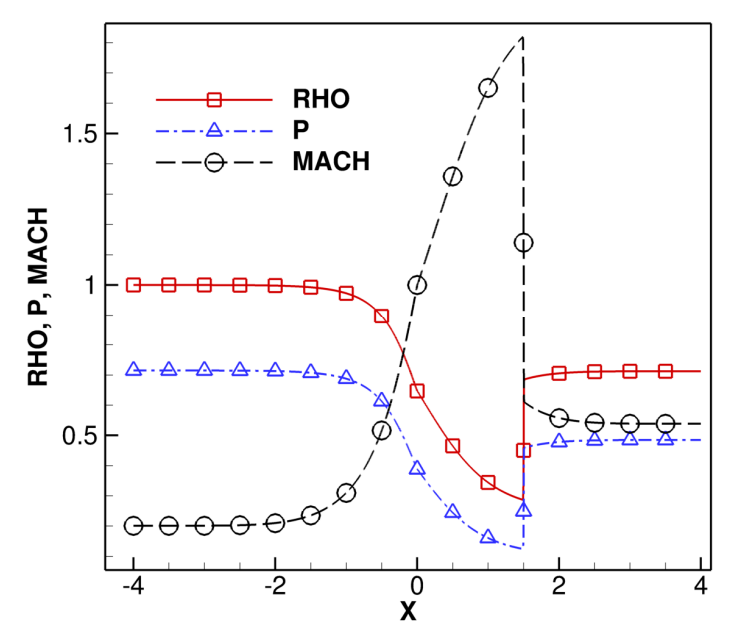

(b)

Fig. 1. Flow fields from the $1 \mathrm{D}$ nozzle flow simulation using $P^{4}$ reconstruction on 1280 elements. (a) Subsonic flow with inlet Mach number 0.2; (b) transonic flow with shock located at $x=1.5$. In this figure, 'RHO' stands for density; 'P' stands for pressure; and 'MACH' stands for Mach number.

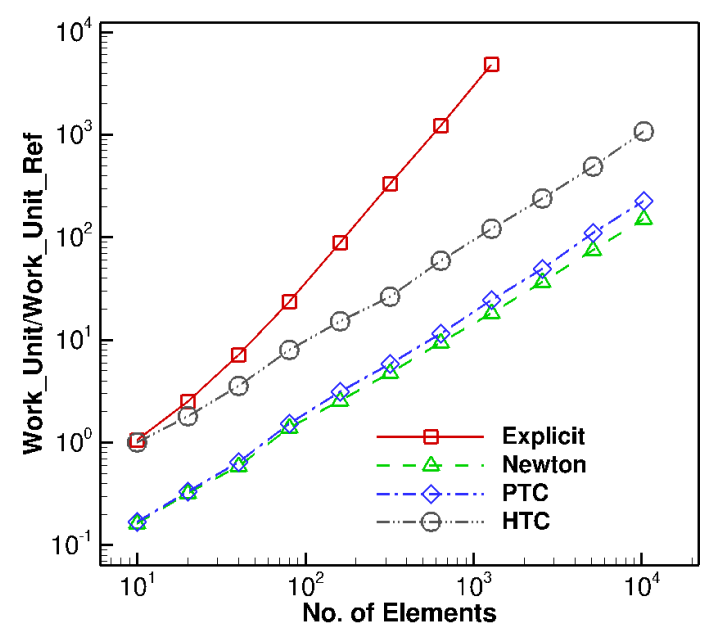

(a)

11 of 17

American Institute of Aeronautics and Astronautics 




(b)

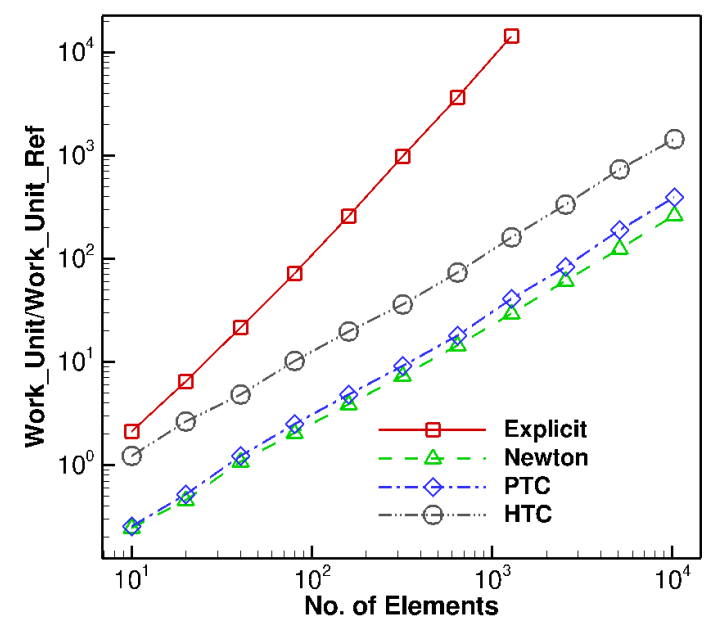

(c)

Fig. 2. Computational cost compasion on different meshes for the $1 \mathrm{D}$ subsonic nozzle flow. (a) $P^{2}$ reconstruction; (b) $P^{3}$ reconstruction; (c) $P^{4}$ reconstruction. In this figure, 'Explicit' indicates the explicit Runge-Kutta method; 'Newton' indicates the Newton method; 'PTC' indicates the pseudo-transient continuation method; 'HTC' indicates the homotopy continuation method.

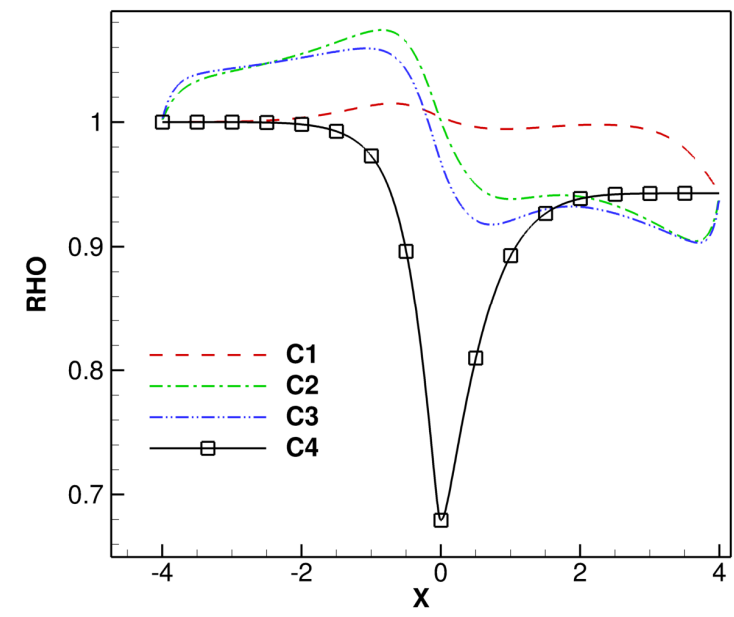

(a)

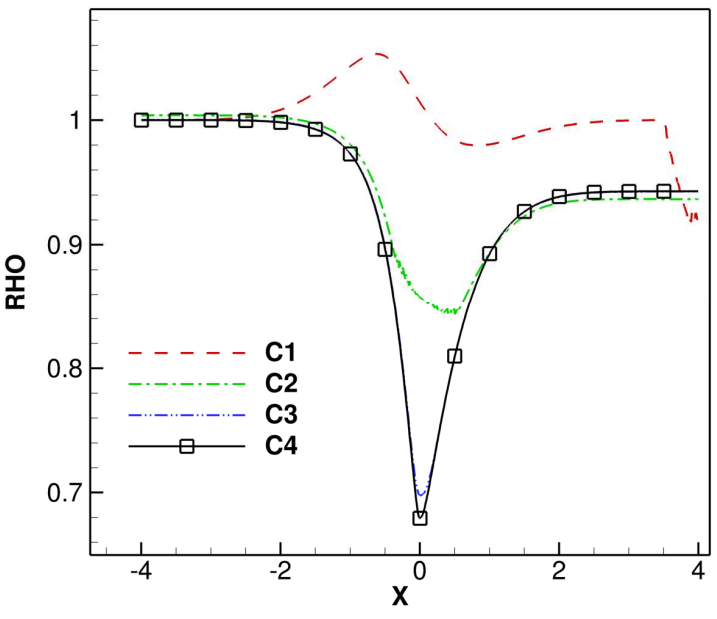

(b)

Fig. 3. The convergence path for the $1 \mathrm{D}$ subsonic nozzle flow using $P^{3}$ reconstruction on 160 elements. (a) Homotopy continuation; (b) pseudo-transient continuation. In this figure, 'C1', 'C2', 'C3' and ' $\mathrm{C} 4$ ' stand for different states during the convergence process. 


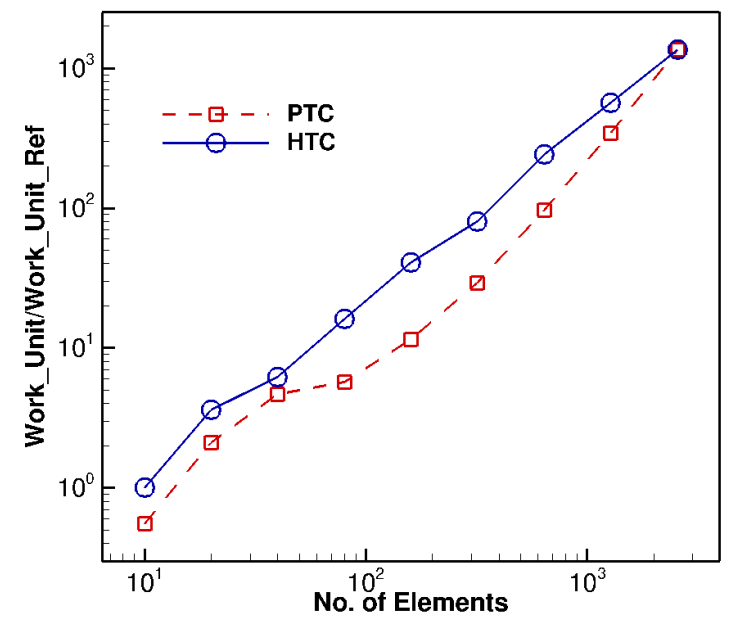

(a)

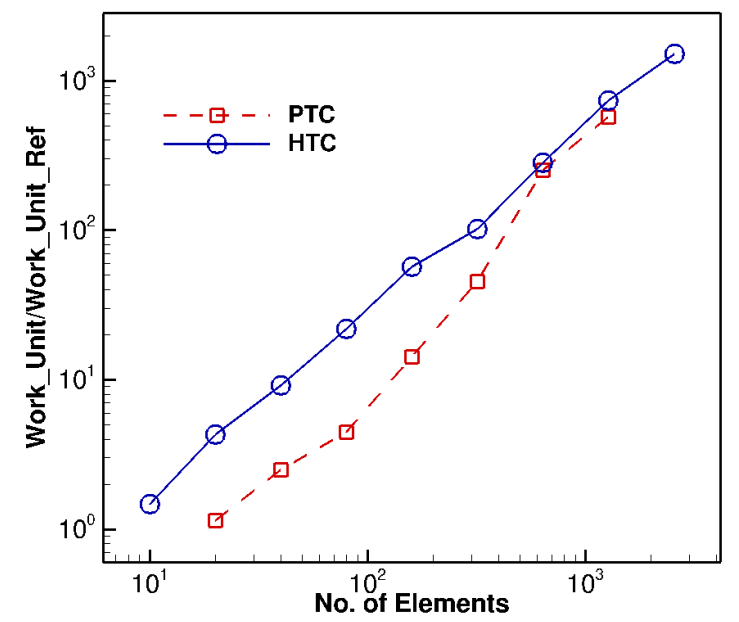

(b)

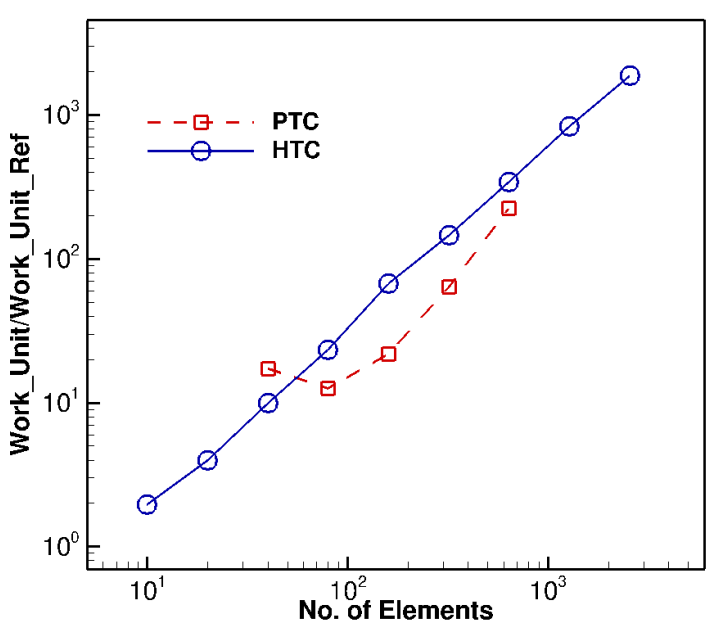

(c)

Fig. 4. Computational cost compasion on different meshes for the $1 \mathrm{D}$ transonic nozzle flow. (a) $P^{2}$ reconstruction; (b) $P^{3}$ reconstruction; (c) $P^{4}$ reconstruction. 


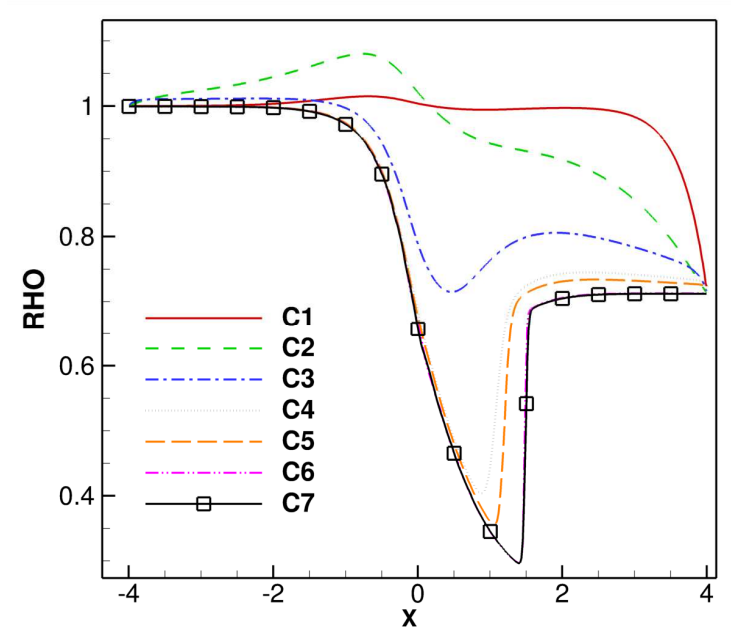

(a)

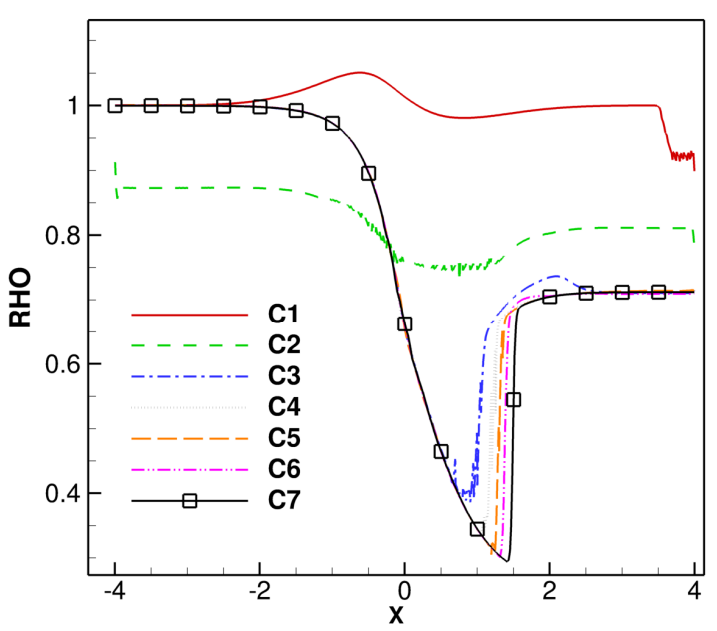

(b)

Fig. 5. The convergence path for the $1 \mathrm{D}$ transonic nozzle flow using $P^{3}$ reconstruction on 160 elements. (a) Homotopy continuation; (b) pseudo-transient continuation.

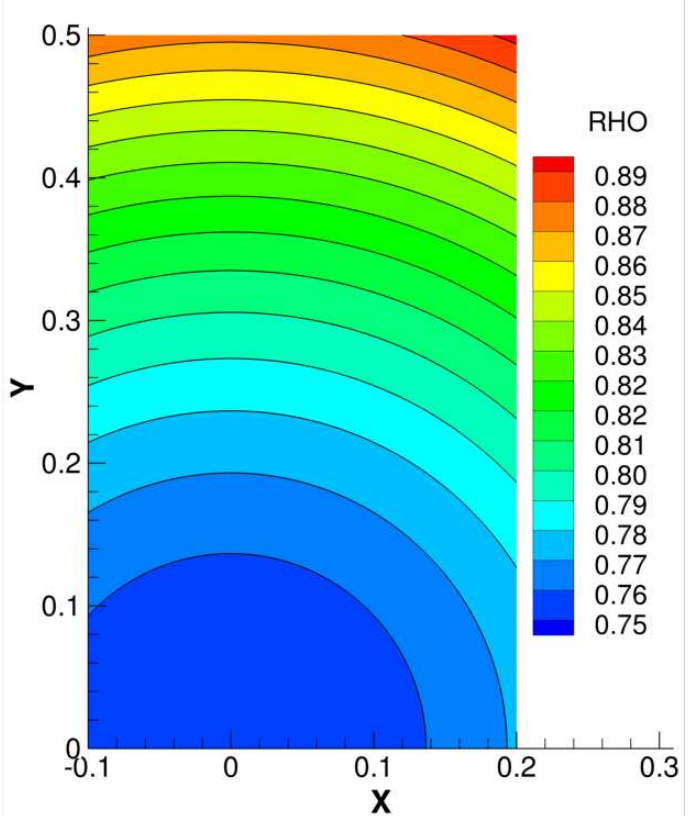

(a)

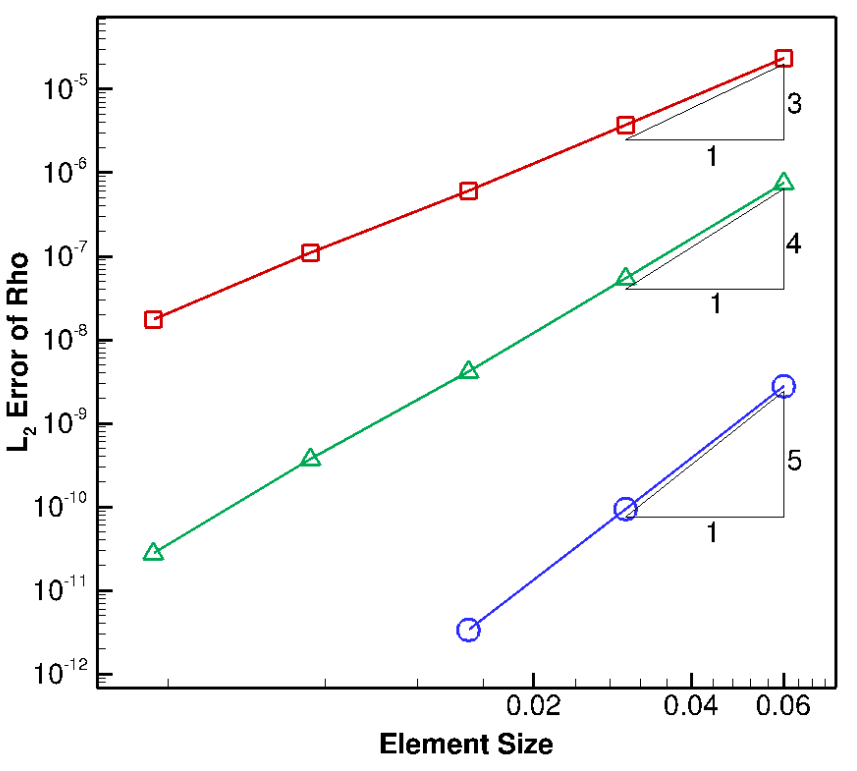

(b)

Fig. 6. (a) Converged solution for the density $\rho$ of the manufactured solution problem using $P^{3}$ reconstruction on a $40 \times 72$ mesh; (b) order of accuracy study using homotopy continuation. 


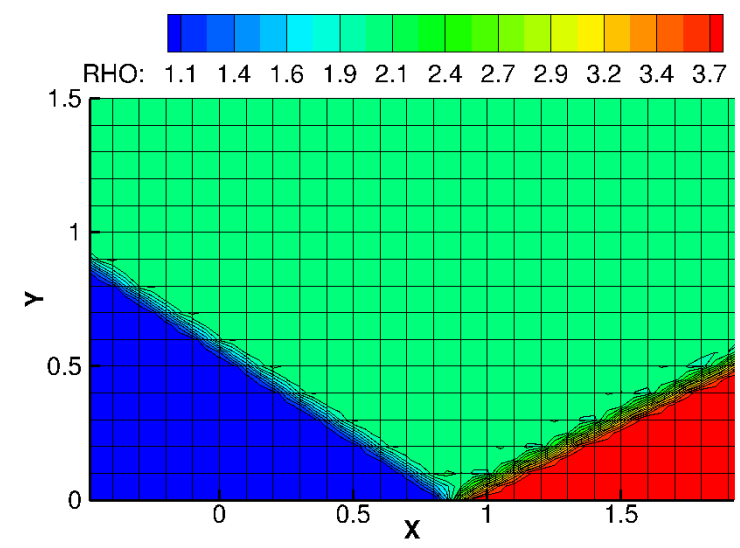

(a)

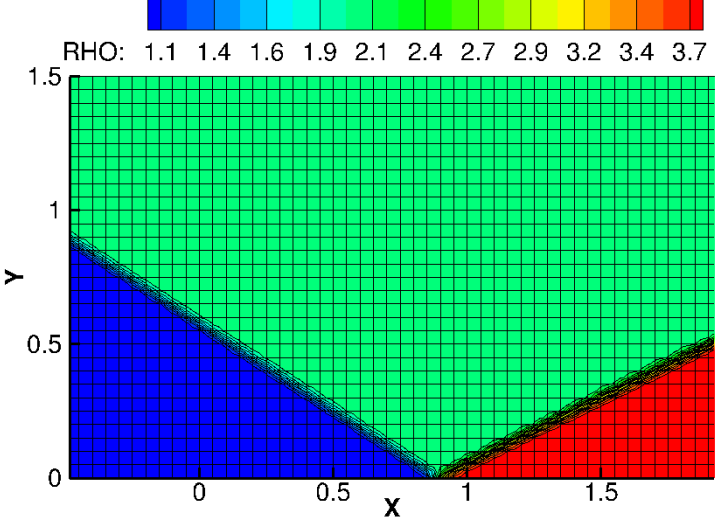

(c)

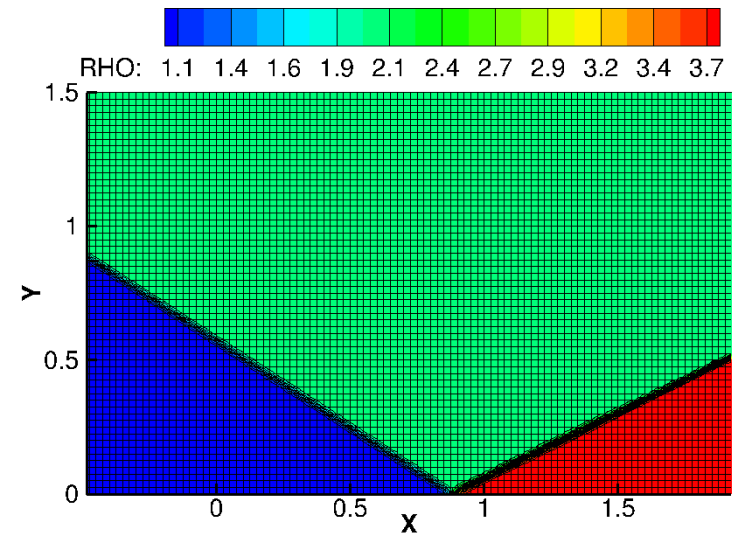

(e)

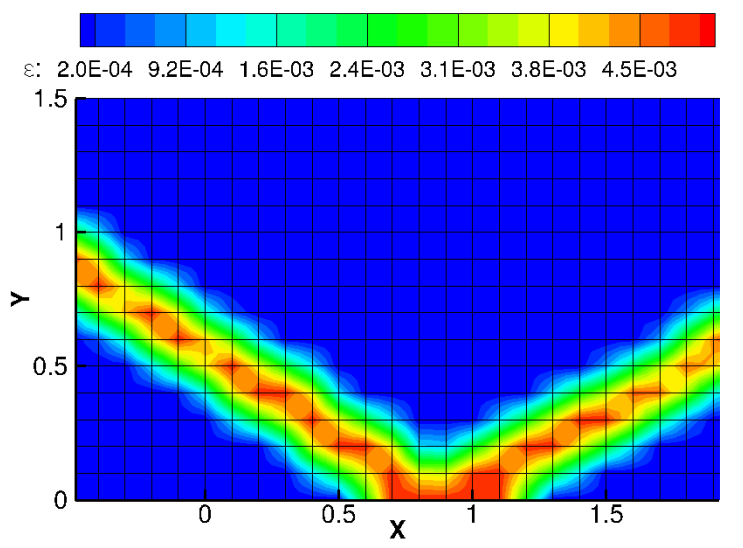

(b)

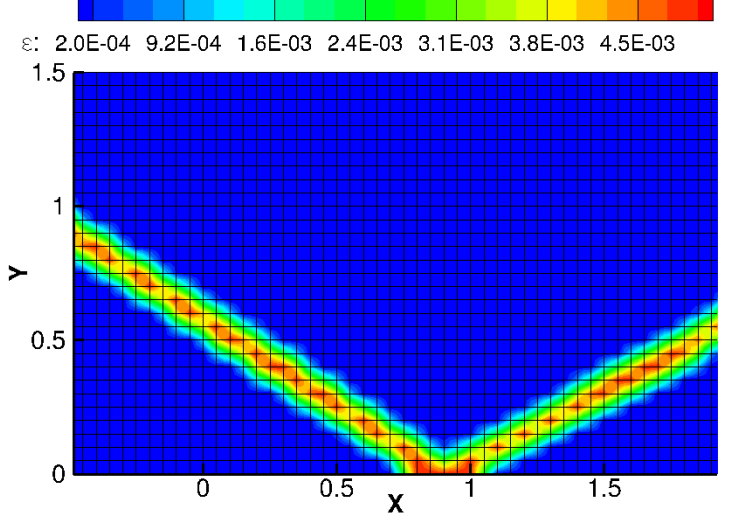

(d)

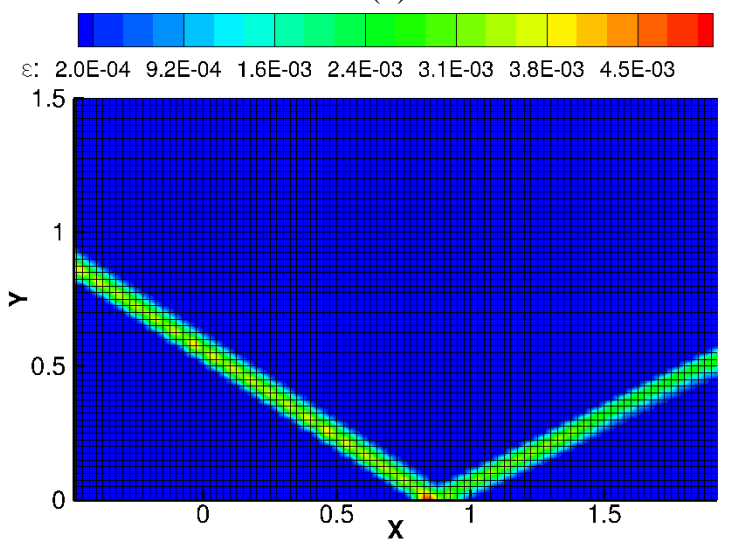

(f)

Fig. 7. Density and artificial viscosity contours of oblique shock reflection using $P^{2}$ reconstruction on three sets of meshes. (a) Density, grid size 1/10; (b) artificial viscosity, grid size 1/10; (c) density, grid size 1/20; (d) artificial viscosity, grid size 1/20; (e) density, grid size 1/40; (f) artificial viscosity, grid size 1/40. 




(a)

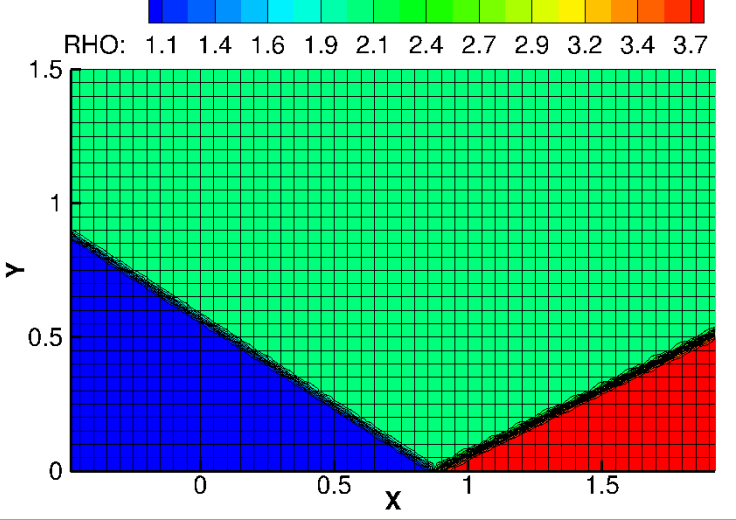

(c)

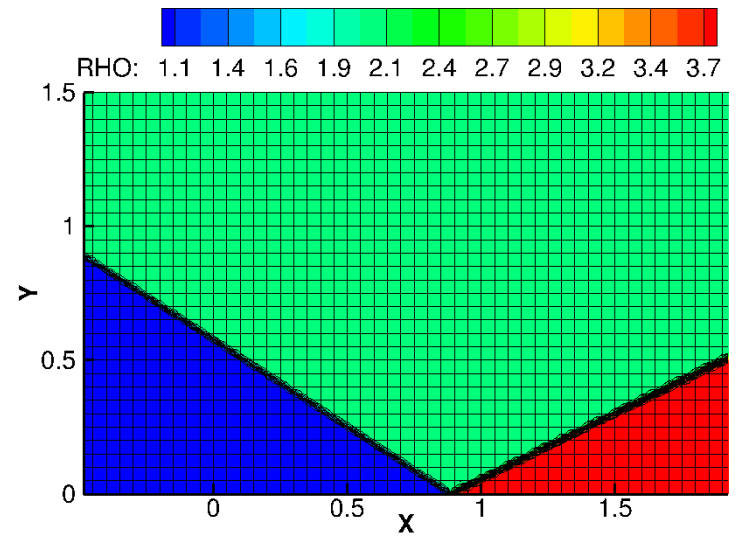

(e)

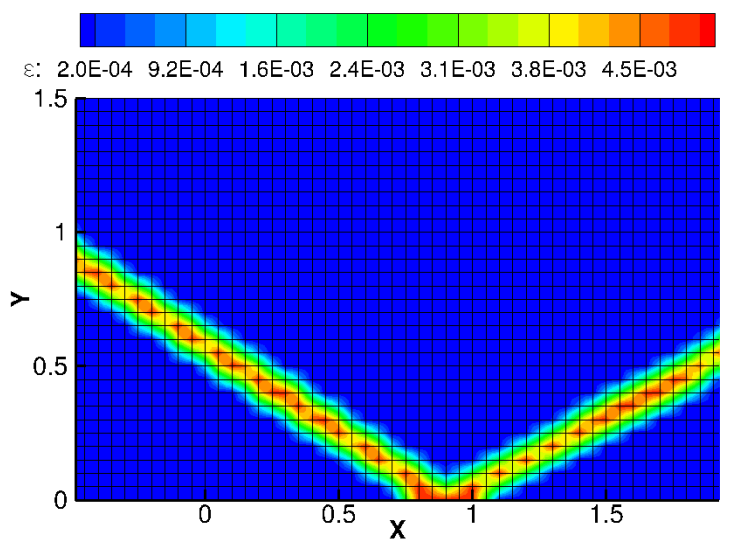

(b)

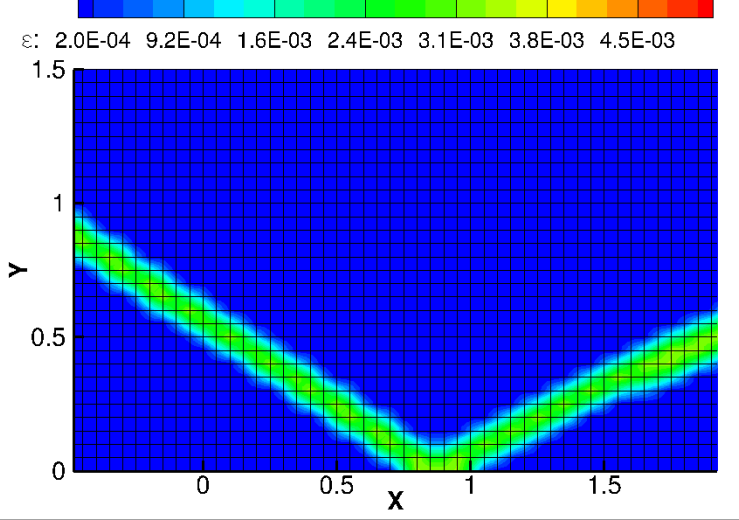

(d)

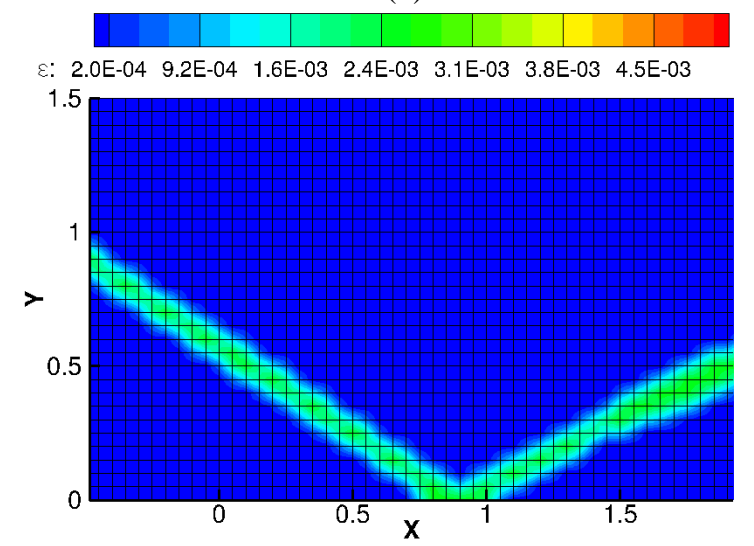

(f)

Fig. 8. Density and artificial viscosity contours of oblique shock reflection using different reconstruction orders on the mesh with characteristic element length of $1 / 20$. (a) Density, $P^{2}$ reconstruction; (b) artificial viscosity, $P^{2}$ reconstruction; (c) density, $P^{3}$ reconstruction; (d) artificial viscosity, $P^{3}$ reconstruction; (e) density, $P^{4}$ reconstruction; (f) artificial viscosity, $P^{4}$ reconstruction. 


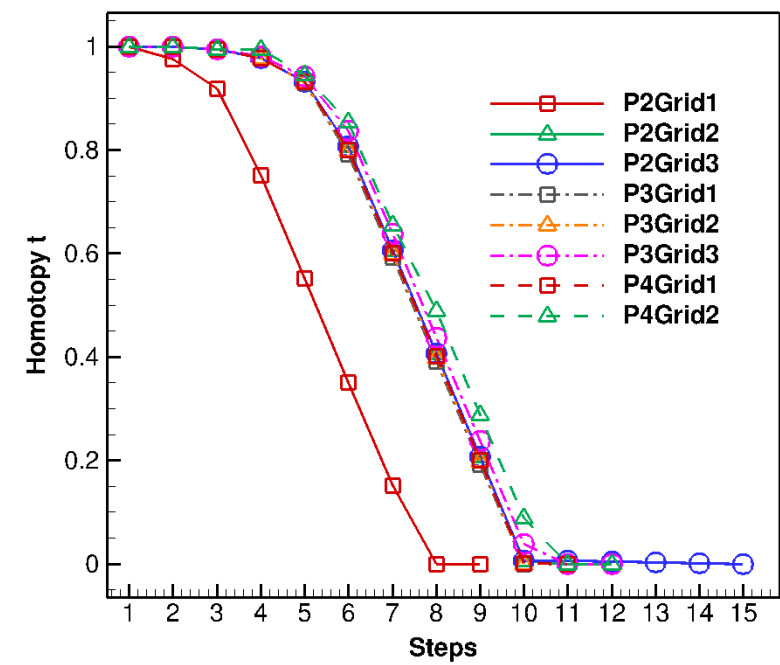

(a)

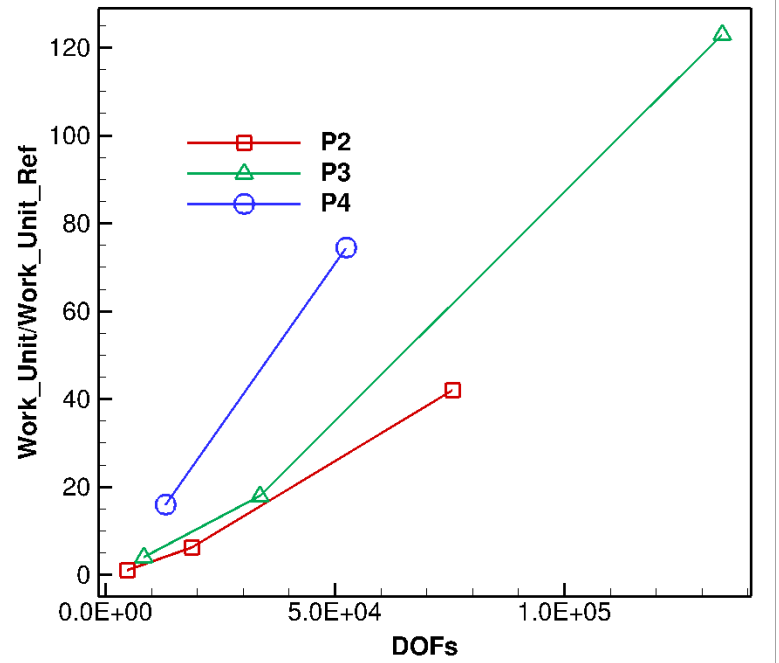

(b)

Fig. 9. (a) Homotopy tracking paths for different reconstruction orders and meshes; (b) normalized work unit versus degrees of freedoms (DOFs) for different orders of reconstruction. In this figure, 'P2', 'P3', and 'P4' indicates $3^{\text {rd }}$, $4^{\text {th }}$, and $5^{\text {th }}$ order of reconstruction respectively; 'Grid1', 'Grid2', and 'Grid3' denotes mesh with characteristic element length of $1 / 10,1 / 20$, and $1 / 40$ respectively. 